\title{
Dynamic response of the tower of a NREL5MW wind turbine generator
}

\author{
Ivo Angelov ${ }^{1, *}$ \\ ${ }^{1}$ Technical University - Sofia, Department of Mechanics, Bulgaria, 8 Kl. Ohridski Blvd., 1000 Sofia, Bulgaria
}

\begin{abstract}
Considering the complexity of the aerodynamic interaction between a non-homogeneous, nonstationary wind field and a wind turbine, one can easily establish that the constructive elements of the turbine will be exposed to dynamic loads, which will eventually lead to forced motion and particularly to forced vibrations. One of the most significant elements of every wind turbine is the tower of the generator. The tower is subjected to forced vibrations and transmits all dynamic loads that appear in the wind turbine. That is why a dynamic analysis of the tower is worth executing. In this research such an analysis is made considering the tower of the generator as Euler-Bernoulli beam structure and considering it as a LoveKirchhoff shell structure.
\end{abstract}

\section{Introduction}

The purpose of this study is to analyse the dynamic behaviour of a tower of a wind turbine generator of high power such as the NREL5MW wind turbine. Considering the aerodynamic interaction between the wind and the whole wind turbine, the station is subjected to dynamic loads. These loads are transmitted to the ground through the tower of the turbine. That is why a dynamic survey on the tower is of significant interest. The dynamic analysis can give the natural characteristics of the tower, can provide more knowledge on the forced vibrations and show a relation between wind speed and generated stresses.

In our previous studies [1] a modified BEM theory was used to obtain the aerodynamic forces acting on the turbine, using a non-homogenous, non-stationary wind field model. The data obtained from these studies will be used in the present one.

The following assumptions are made:

- The pitch angle of the blades is assumed to be constant and an active pitch control system is not considered;

- Any deformation of the blades as well as their mass and inertial characteristics are neglected;

- The wind field is considered non-homogenous, nonstationary.

\section{A dynamic model of the tower of NREL5MW wind turbine}

\subsection{Geometrical and material properties of the tower}

The tower of NREL5MW [2] is a truncated cone shown in Fig. 1. The geometric and material characteristics are given in Table 1.

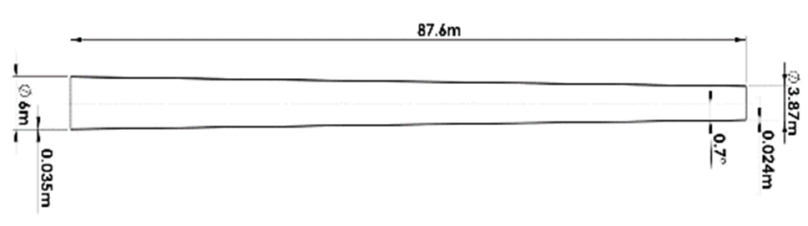

Fig. 1. NREL5MW Tower.

Table 1. Geometric and material characteristics of the tower of the NREL5MW wind turbine.

\begin{tabular}{|c|c|c|c|}
\hline \multicolumn{2}{|c|}{ Geometric characteristics } & \multicolumn{2}{|c|}{ Characteristics of the material } \\
\hline Height, $\mathrm{m}$ & 87,6 & Density, kg.m ${ }^{-3}$ & 8500 \\
\hline Cone angle, $^{\circ}$ & 1,4 & Mass, kg & 347460 \\
\hline $\begin{array}{c}\text { Outer diameter } \\
\text { at the base, } \mathrm{m}\end{array}$ & 6 & Young's module, GPa & 210 \\
\hline $\begin{array}{c}\text { Inner diameter } \\
\text { at the base, } \mathrm{m}\end{array}$ & 5,93 & Shear module, GPa & 80,8 \\
\hline $\begin{array}{c}\text { Outer diameter } \\
\text { at the top, } \mathrm{m}\end{array}$ & 3,87 & Poisson's ration & 0,43 \\
\hline $\begin{array}{c}\text { Inner diameter } \\
\text { at the top, } \mathrm{m}\end{array}$ & 3,82 & Damping ratio, $\%$ & 1 \\
\hline
\end{tabular}

\subsection{External loads}

The tower of the wind turbine transmits almost all forces that appear in the wind turbine generator structure. Three external forces are taken into consideration in the present study. These are the thrust force $F_{t h}$, and the torque $M_{w t}$, both resulting from the aerodynamic interaction between the wind and the wind turbine [1] and a non-uniform distribution acting on the tower, resulting from the interaction between the wind and the tower itself - Fig. 2.

The determination of these forces was the subject of previous studies [3]. The purpose here is to derive an analytical form of the external loads. Using [1] one can

\footnotetext{
* Corresponding author: ivvoangelov@,gmail.com
} 
obtain a dependence between $F_{t h}$ and $M_{w t}$ and the wind speed, defining them as a function of the wind speed $V_{\infty}$ as shown in Fig. 3.

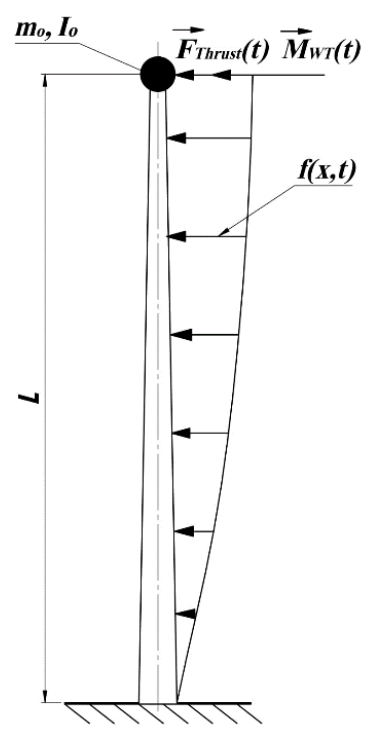

Fig. 2. Acting forces on the tower of NREL5MW.
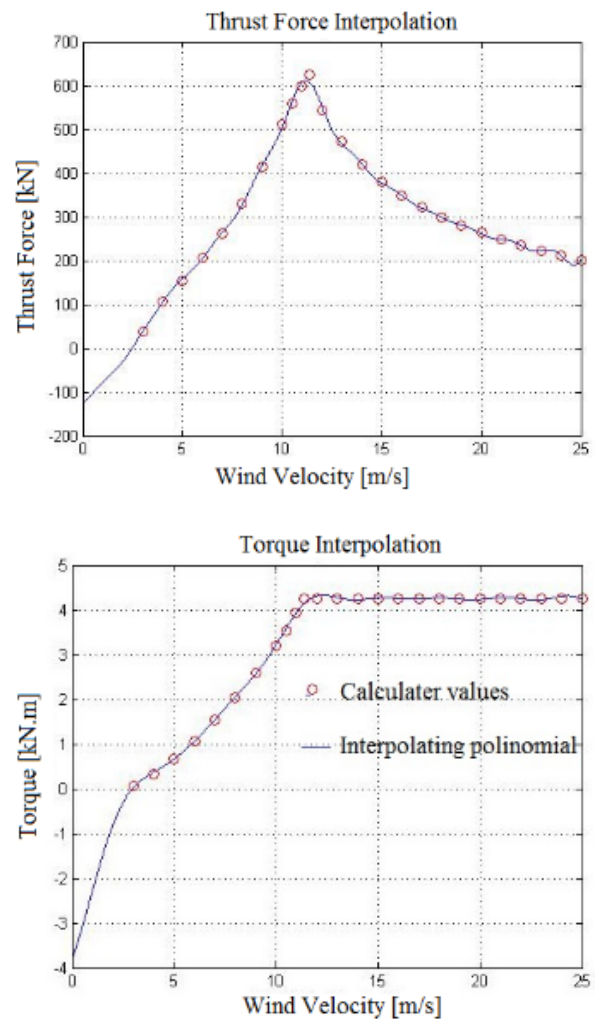

Fig. 3. Thrust force and torque as a function of the wind speed.

In order to obtain these dependencies into an analytical form a polynomial aproximation is made using the discrete values. The aproximation is in polynomial form:

$$
\begin{gathered}
F_{t h}\left(V_{\infty}\right)=\sum_{m=1}^{M} a_{m} V_{\infty}^{m} \\
M_{W T}\left(V_{\infty}\right)=\sum_{m=1}^{M} b_{m} V_{\infty}^{m},
\end{gathered}
$$

where:

$a_{m}$ and $b_{m}$ are coefficients to be defined;
$V_{\infty}$ was derived in analytical form decomposing the power spectrum density function $S_{u}$, in Shinozuka series [4]:

$$
V_{\infty}=\sqrt{2} \sum_{i=0}^{N-1} \sqrt{S_{u^{\prime}}(f) \Delta f} \cos \left(2 \pi f_{i} t+f_{i}\right),
$$

where:

$\Delta f$-frequency summation step;

$f_{i}$ - random phases.

Graphical visualization of (2) is shown in Fig.4.

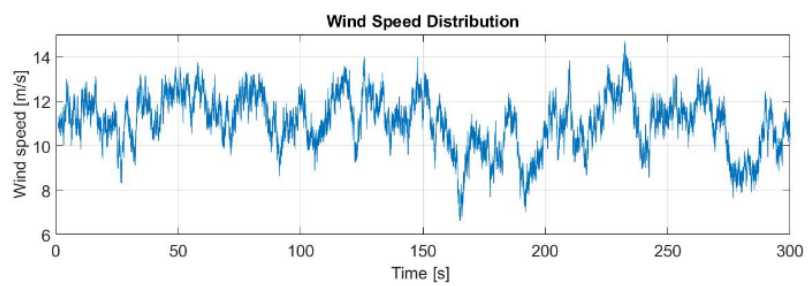

Fig. 4. Wind speed velocity distributed in time.

Substituting (2) in (1) gives an expresion of the thrust force $F_{t h}(t)$ and the torque $M_{w t}(t)$ as a function of time. The obtained fuctions are shown in Fig. 5.
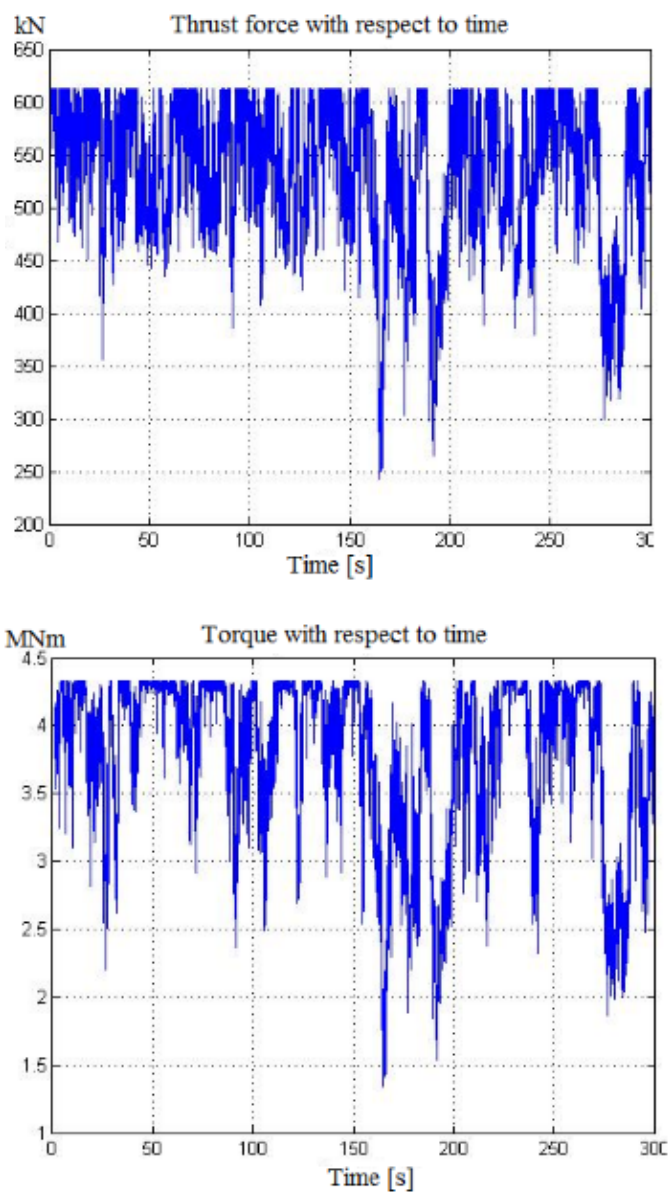

Fig. 5. Thrust force $F_{t h}(t)$ and torque $M_{w t}(t)$ as a function of time.

The distributed load is defined similarly with the difference that one more function is required to represent the change in magnitude with respect to the height of the tower $f(x, t)=X(x) F\left(V_{\infty}(t)\right)$, where $X(x)$ represents the vertical wind speed distribution [1]. Fig. 6 shows the distributed load $f(x, t)$. 


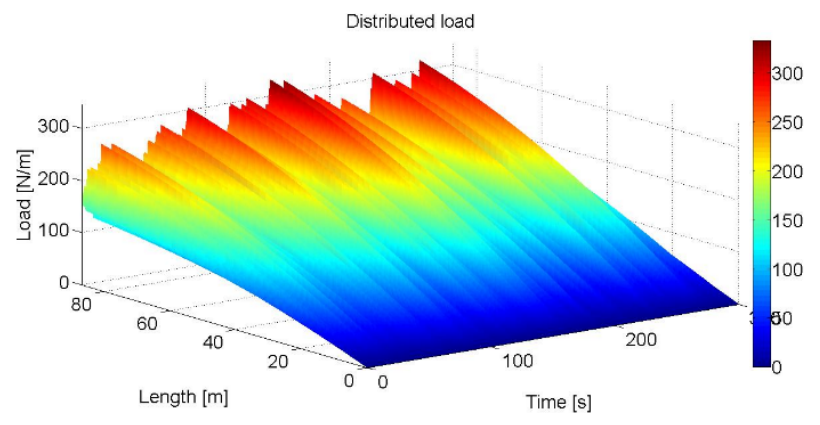

Fig. 6. Non-uniform distributed load as a function of time and height $f(x, t)$.

The boundary conditions of the model are as follows: a fixed end at the base of the tower and a point mass $m_{0}$ and inertia moment $I_{0}$ (with respect to the axis of the generator) at the top end - Fig.2. This point mass and inertia represent the mass characteristics of all bodies attached to the tower, such as wind wurbine, electric generator, hub, nacelle, shafts, bearings, etc.

\subsection{Dynamic analysis of the tower in terms of the Euler-Bernoulli beam theory}

In this section a dynamic analysis of the tower is executed using Euler-Bernoulli beam theory with equation in general form:

$$
\frac{\partial^{2}}{\partial x^{2}}\left[E I(x) \frac{\partial^{2} w(x, t)}{\partial x^{2}}\right]+\rho A(x) \frac{\partial^{2} w(x, t)}{\partial t^{2}}=q(x, t),
$$

where:

- $E$ - Young's modulus of the tower material;

- $I(x)$ - geometric moment of inertia of th tower;

- $w$ - transverse displacement;

- $\rho-$ density of the material of the tower's material;

- $q(x, t)$ - distributed load.

The implemented methodology is as follows. First, the natural frequencies $\omega_{i}$ are determined using Rayleigh-Ritz formula [5]:

$$
\omega_{i}^{2}=\frac{\int_{0}^{L} E I(x)\left[\frac{d^{2} W_{i}^{*}(x)}{d x^{2}}\right]^{2} d x}{\int_{0}^{L} \rho A(x)\left[W_{i}^{*}(x)\right]^{2} d x+m_{0}\left[W_{i}^{*}(L)\right]^{2}+I_{0}\left[\frac{d W_{i}^{*}(L)}{d x}\right]^{2}},
$$

where $W_{i}^{*}(x)$ are aproximated functions, called Ansatzfunktion. For approximated functions are used the exact mode shapes of an uniform beam, with the same boundaries, the same length, the same material and mass properties, The mode shapes of this simplified beam model are shown in Fig. 7.

Substituting the aproximated mode shapes in (4) gives the corresponding frequencies, which are shown in Table 2.

Table 2. Natural frequencies of the tower of NREL5MW wind turbine with boundaries point mass and inertia at top end.

\begin{tabular}{|c|r|}
\hline$\omega_{1}, \mathrm{~Hz}$ & 0,3475 \\
\hline$\omega_{2}, \mathrm{~Hz}$ & 3,3234 \\
\hline$\omega_{3}, \mathrm{~Hz}$ & 11,0003 \\
\hline$\omega_{4}, \mathrm{~Hz}$ & 22,2794 \\
\hline$\omega_{5}, \mathrm{~Hz}$ & 37,0453 \\
\hline
\end{tabular}

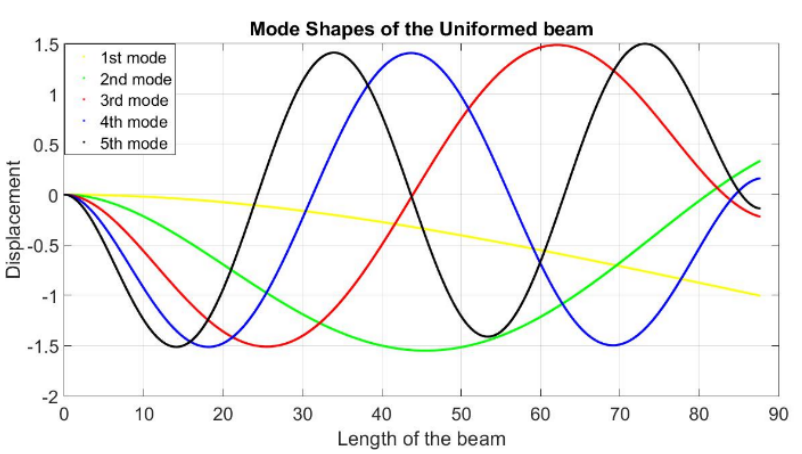

Fig. 7. First five mode shapes of the simplified beam.

Once the natural frequencies are obtained the partial differential Euler-Bernoulli equation (3) becomes a homogenous, ordinary differential equation ordinary with non-constant coefficions.

$$
E I(x) \frac{d^{4} W_{i}(x)}{d x^{4}}+\rho A(x) \omega_{i}^{2} W_{i}(x)=0
$$

It will be solved in order to determine the mode shapes of the tower. Equation (5) does not have an exact solution, Here it is solved numerically in matlab using "bvp4c" solver [6]. The obtained solution is shown in Fig.8.

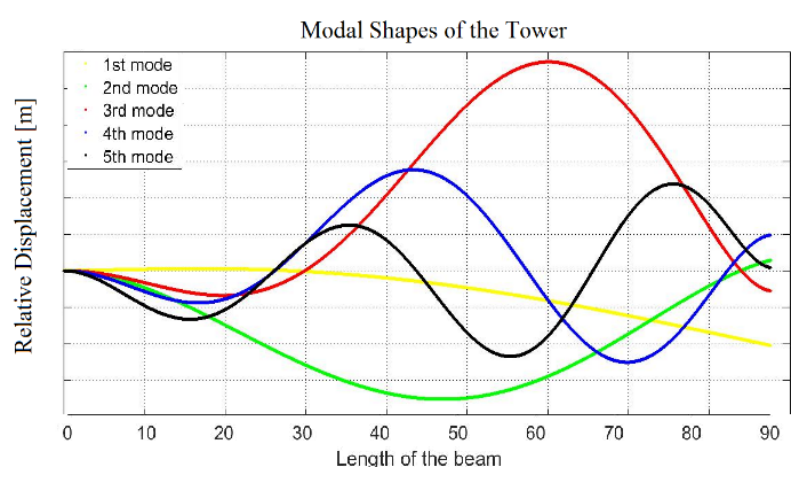

Fig.8. First five mode shapes of the tower of NREL5MW .

Once the natural characteristics of the tower are defined, they can be used to determine the response of the structure. For this purpose a modal analysis aproach is used [7]. The response

$$
w(x, t)=\sum_{i=1}^{5} W_{i}(x) \mu_{i}(t)
$$

is defined as a sum of the products of the mode shapes $W_{i}(x)$ and a time dependence function $\mu_{i}(t)$ :

$$
\mu_{i}(t)=\frac{1}{\omega_{i}} \int_{0}^{t} Q(\tau) \sin \left[\omega_{i}(t-\tau)\right] d \tau
$$

where

$$
Q_{i}(t)=\int_{0}^{L} W_{i}(x) f(x, t) d x .
$$

In order for the solution to show both qualitative and quantitative characteristics, the mode shapes must be normalized, using the following scaling coefficient $C_{i}$.

$$
C_{i} \int_{0}^{L} \rho A(x) W_{i}^{2}(x) d x=1
$$

The modal analysis solution shows the participation of each mode shape in the beam response. The described 
methodology is executed in Matlab and gives us the following results. Fig. 9 shows the response due to the distributed load $f(x, t)$ separated into the first four mode shapes. It is observed that the solution is dominated by the first mode. Every subsequent mode participates less as compared to the previous one. Fig. 10 shows the response due to the thrust force $F_{t h}$. Fig. 11 shows the response due to the torque $M_{w t}$. Fig. 12 shows the total response of the tower due to all the external excitations. Fig. 13 shows the normal stresses, generated in the structure due to the external loads.
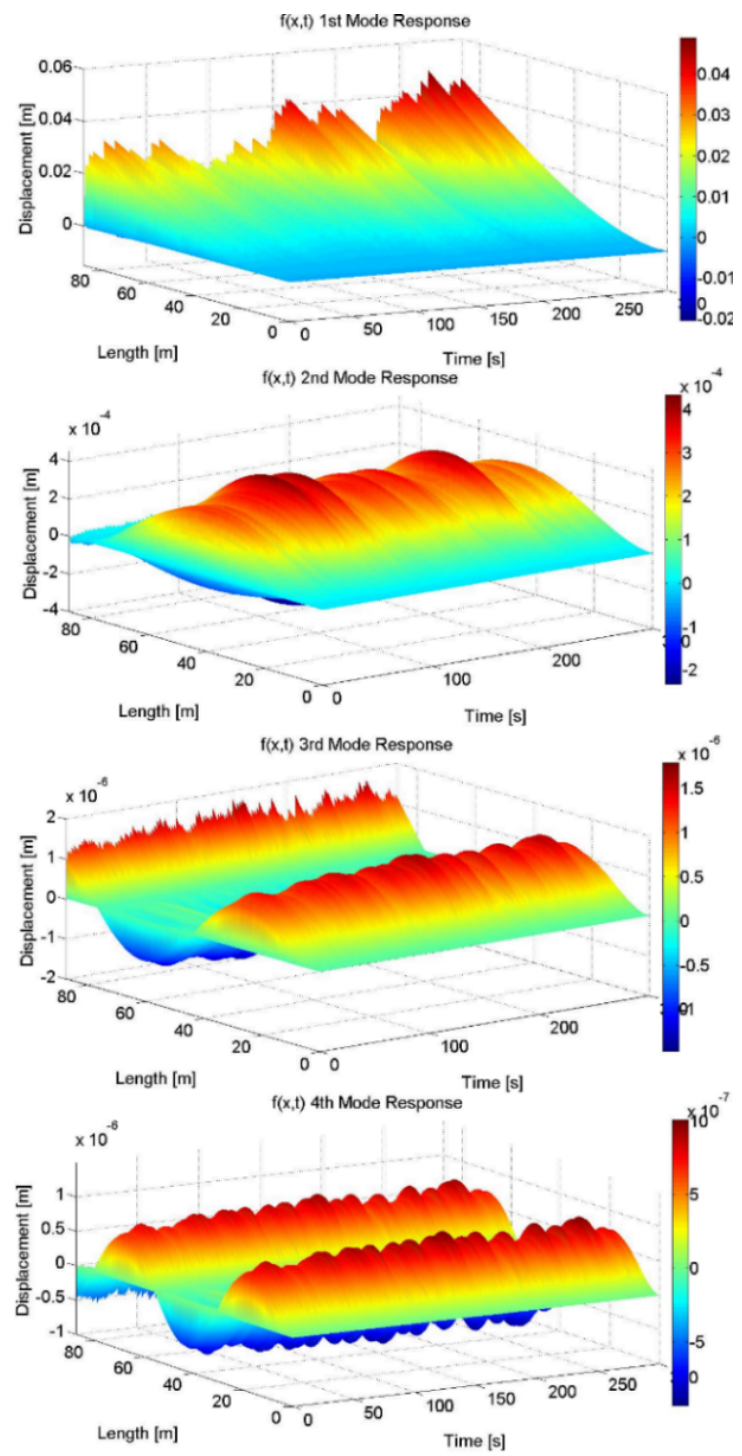

Fig. 9. Beam response due to the distributed load $f(x, t)$ with the exact participation of the first 4 mode shapes.

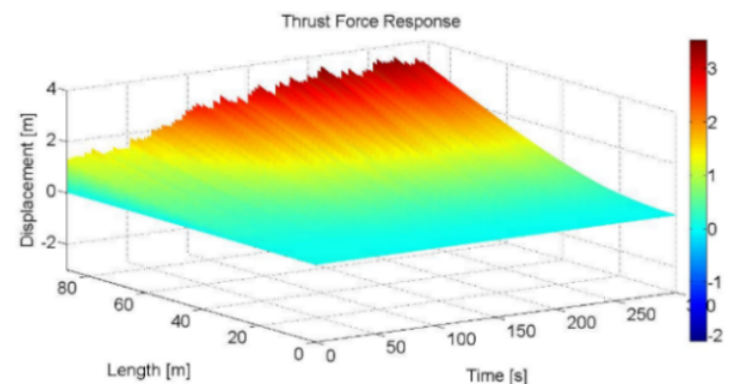

Fig. 10. Beam response due to the thrust force $F_{t h}$.

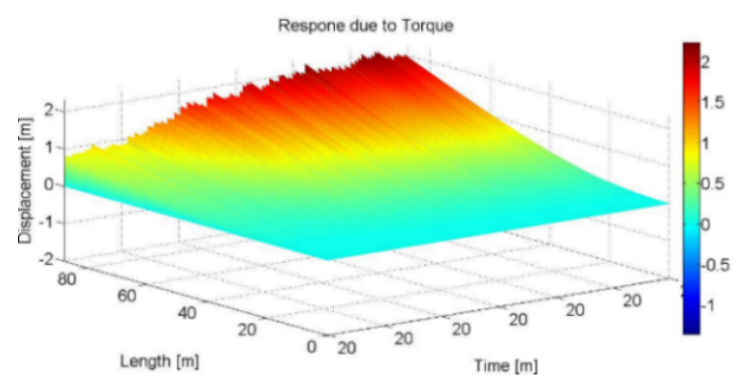

Fig. 11. Beam response due to the torque $M_{w t}$.
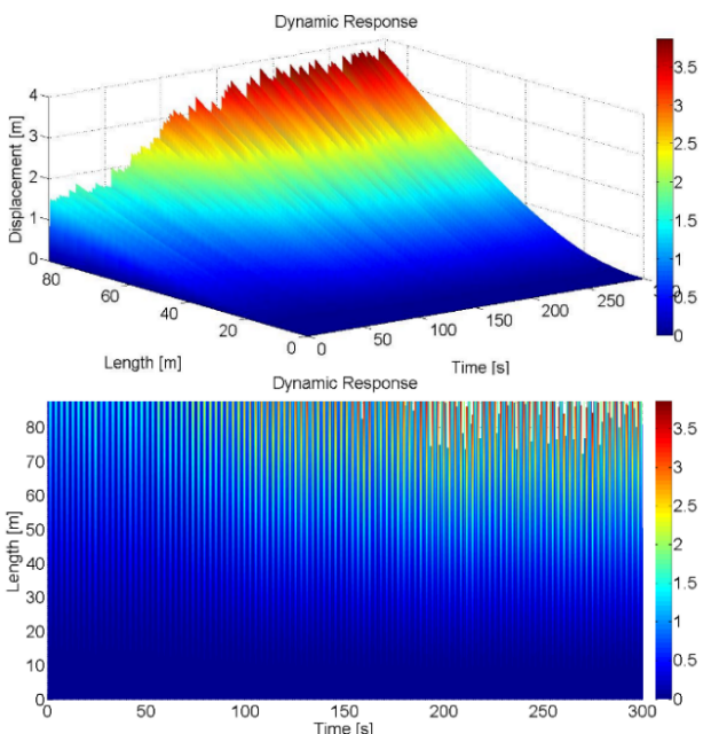

Fig. 12. Beam response due to all external loads.
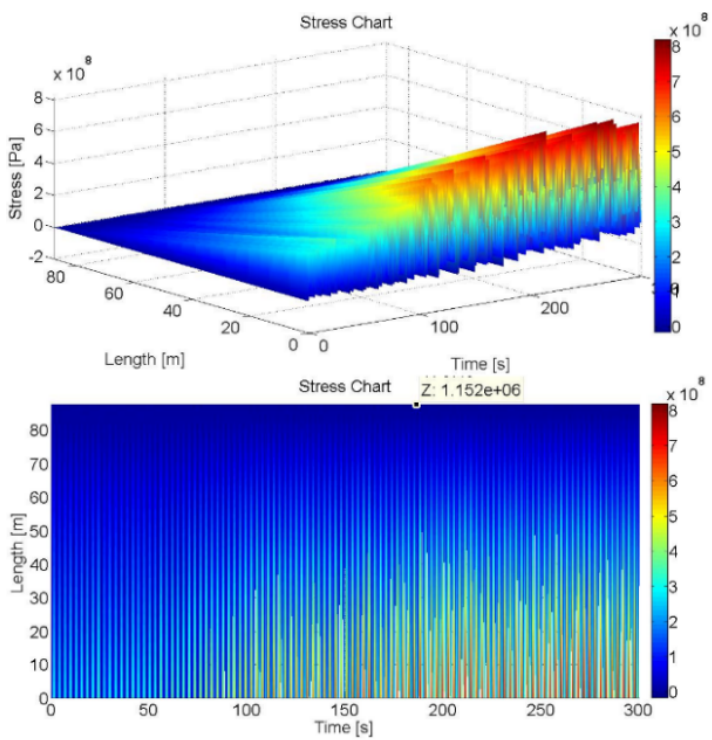

Fig. 13. Generated normal stresses due to all external loads.

\subsection{Numerical analysis in ANSYS Workbench}

The modal numerical survey made in ANSYS Workbech shows that the tower of NREL5MW has the behavior of shell structures. It shows the typical for a shell structure circumferential mode shapes. It also shows a difference in the natural frequencies corresponding to the shell bending mode shapes. The comparison between the frequencies is shown in Table 3. 
Table 3. Natural frequencies of the structure, corresponding to the bending mode shapes.

\begin{tabular}{|c|c|c|}
\hline $\begin{array}{c}\text { Natural } \\
\text { frequencies }\end{array}$ & $\begin{array}{c}\text { Euler-Bernoulli } \\
\text { beam analysis }\end{array}$ & $\begin{array}{c}\text { ANSYS numerical } \\
\text { results }\end{array}$ \\
\hline$\omega_{1}, \mathrm{~Hz}$ & 0,3475 & 0,331 \\
\hline$\omega_{2}, \mathrm{~Hz}$ & 3,3234 & 1,967 \\
\hline$\omega_{3}, \mathrm{~Hz}$ & 11,0003 & 4,61 \\
\hline$\omega_{4}, \mathrm{~Hz}$ & 22,2794 & 10,531 \\
\hline$\omega_{5}, \mathrm{~Hz}$ & 37,0453 & 18,874 \\
\hline
\end{tabular}

Regardless of this difference, the performed analysis in section 2.3 is applicable for all kinds of continuous structures. Considering that the response is dominated by the first mode shape a significant difference in the solution will not appear. That is because there is only a slight difference in the frequency corresponding to the first mode.

\subsection{Natural characteristics of a shell structure tower model}

In order to confirm the shell behavior of the tower a shell model is analyzed in this section. For easier analysis and determination of the natural characteristics, the model of the tower is simplified as follows. It is considered uniform with a constant-circular cross section and constant thickness. The top end is set free without point mass and inertia. Fig. 14.

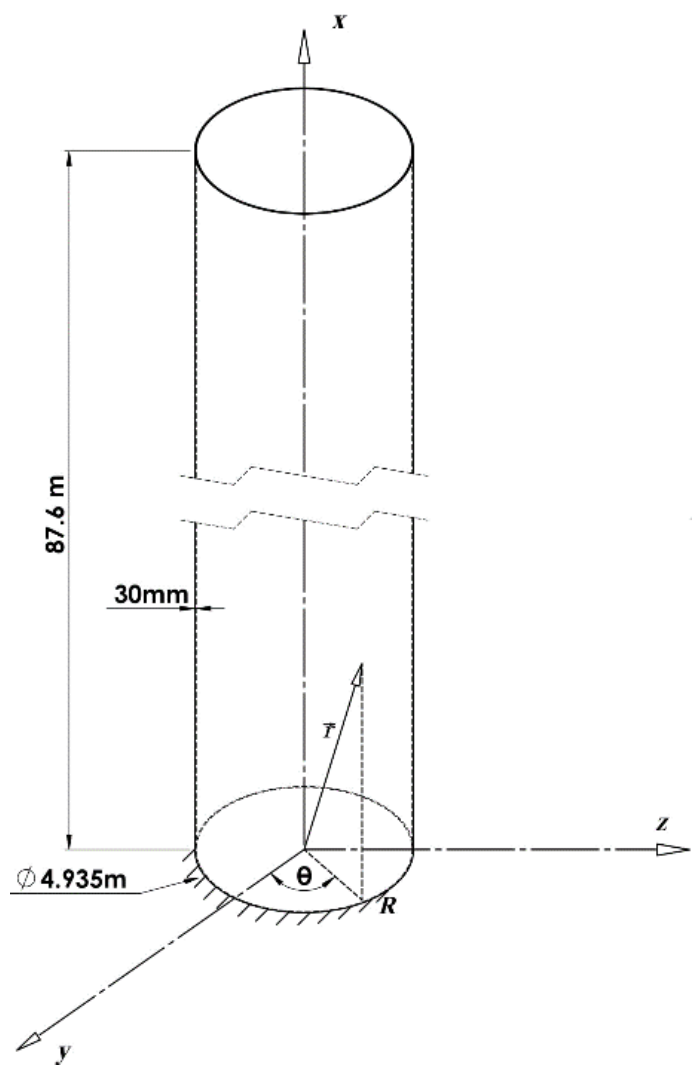

Fig. 14. Shell structure model. $R$ and $\theta$ are polar coordinates.

The differential equations known as Love-Kirchhoff equations [6] describing the dynamic behaviour of a shell structure are:
$\left\{\begin{array}{l}-\frac{\partial\left(N_{\alpha \alpha}\right)}{\partial \alpha}-\frac{\partial\left(N_{\beta \alpha}\right)}{\partial \beta}-N_{\alpha \beta} \frac{\partial \mathrm{A}}{\partial \beta}+N_{\beta \beta} \frac{\partial B}{\partial \alpha}-A B \frac{Q_{\alpha z}}{R_{\alpha}}+A B \rho h \ddot{u}=A B q_{\alpha} \\ -\frac{\partial\left(N_{\alpha \beta}\right)}{\partial \alpha}-\frac{\partial\left(N_{\beta \beta}\right)}{\partial \beta}-N_{\beta \alpha} \frac{\partial \mathrm{A}}{\partial \beta}+N_{\alpha \alpha} \frac{\partial B}{\partial \alpha}-A B \frac{Q_{\beta z}}{R_{\beta}}+A B \rho h \ddot{v}=A B q_{\beta} \\ -\frac{\partial\left(Q_{\alpha z} B\right)}{\partial \alpha}-\frac{\partial\left(Q_{\beta z} A\right)}{\partial \beta}+A B\left(\frac{N_{\alpha \alpha}}{R_{\alpha}}+\frac{N_{\beta \beta}}{R_{\beta}}\right)+A B \ddot{w}=A B q_{z} \\ \frac{\partial\left(M_{\alpha \alpha} B\right)}{\partial \alpha}+\frac{\partial\left(M_{\beta \alpha} A\right)}{\partial \beta}+M_{\alpha \beta} \frac{\partial \mathrm{A}}{\partial \beta}-M_{\beta \beta} \frac{\partial B}{\partial \alpha}-Q_{\alpha z} A B=0 \\ \frac{\partial\left(M_{\alpha \beta} B\right)}{\partial \alpha}+\frac{\partial\left(M_{\beta \beta} A\right)}{\partial \beta}+M_{\alpha \beta} \frac{\partial \mathrm{B}}{\partial \alpha}-M_{\alpha \alpha} \frac{\partial A}{\partial \beta}-Q_{\beta z} A B=0\end{array}\right.$

where:

- the $\alpha, \beta$-curvilinear coordinates;

- $A, B$ - lengths of the radius vector projection respectively on $\alpha, \beta$ coordinate;

- $R_{\alpha}, R_{\beta}$ - radiuses of the curvilinear coordinates

- z - linear coordinate;

- $N_{i j}, Q_{i j}$ - normal and tangential forces per unit length

- $M_{i j}$ - moments of the forces

- $R_{i}$ - radiuses of curvature;

After a simplification for a cylindrical shell structure equations (10) take the form of:

$\mid C\left(\frac{\partial^{2} u}{\partial x^{2}}+\frac{1-v}{2 R^{2}} \frac{\partial^{2} u}{\partial \theta^{2}}+\frac{v}{R} \frac{\partial w}{\partial x}+\frac{1+v}{2 R} \frac{\partial^{2} v}{\partial x \partial \theta}\right)+q_{x}=\rho h \frac{\partial^{2} u}{\partial t^{2}}$

$\mid C\left(\frac{1-v}{2} \frac{\partial^{2} v}{\partial x^{2}}+\frac{1}{R^{2}} \frac{\partial^{2} v}{\partial \theta^{2}}+\frac{1}{R^{2}} \frac{\partial w}{\partial \theta}+\frac{1+v}{2 R} \frac{\partial^{2} u}{\partial x \partial \theta}\right)+$

$\mid D\left(\frac{1-v}{2 R^{2}} \frac{\partial^{2} v}{\partial x^{2}}+\frac{1}{R^{4}} \frac{\partial^{2} v}{\partial \theta^{2}}-\frac{1}{R^{4}} \frac{\partial^{3} w}{\partial \theta^{3}}-\frac{1}{R^{2}} \frac{\partial^{3} w}{\partial x^{2} \partial \theta}\right)+q_{\theta}=\rho h \frac{\partial^{2} v}{\partial t^{2}}$

$\mid D\left(\frac{\partial^{4} w}{\partial x^{4}}+\frac{1}{R^{2}} \frac{\partial^{3} v}{\partial x^{2} \partial \theta}-\frac{2}{R^{2}} \frac{\partial^{4} w}{\partial x^{2} \partial \theta^{2}}-\frac{1}{R^{4}} \frac{\partial^{4} w}{\partial \theta^{4}}+\frac{1}{R^{4}} \frac{\partial^{3} v}{\partial \theta^{3}}\right)-$

$\mid C\left(\frac{1}{R^{2}} \frac{\partial v}{\partial \theta}+\frac{w}{R^{2}}+\frac{v}{R} \frac{\partial u}{\partial x}\right)+q_{z}=\rho h \frac{\partial^{2} w}{\partial t^{2}}$

known as Donnell-Mushtari-Vlasov Theory [7, 8], where:

- $w, v, u$ - displacements respectively in transverse, circumferential and axial direction;

- $q_{x}, q_{\theta}, q_{z}$ are projection of the distributed load;

- C and D - constants depending on material's properties;

- $v$ - Poisson's ratio of the material;

- $h$ - thickness of the shell.

The system of PDE (11) does not have an exact solution. The natural frequencies corresponding to the shell bending mode shapes are determined using a semianalytical analysis based on Rayleigh-Ritz approach [9, 10]. The idea is that the solution of (11) is approximated in the following form:

$\mid u(x, \theta, t)=\left[A_{1} \frac{d W_{1}(x)}{d x}+A_{2} \frac{d W_{2}(x)}{d x}\right] \cos (n \theta) \sin (\omega t) ;$
$\mid v(x, \theta, t)=\left[B_{1} W_{1}(x)+B_{2} W_{2}(x)\right] \sin (n \theta) \sin (\omega t) ;$
$\mid w(x, \theta, t)=\left[C_{1} W_{1}(x)+C_{2} W_{2}(x)\right] \cos (n \theta) \sin (\omega t)$,

where $W_{1}(x)$ are the beam bending modes for a clampedfree boundaries and $W_{2}(x)$ are the beam bending modes for a clamped-pinned boundaries. The described procedure is executed in Matlab. The bending modes of a shell structure are these for $n=1$ and $m=1,2,3 \ldots$ A comparison between the frequencies corresponding to the first five bending modes of the described structure considered as a beam, as a shell and results from ANSYS are shown in Table 4. 
Table 4. Natural frequencies corresponding to the bending mode shapes of the model considered as a beam structure, as a shell structure and numerical results.

\begin{tabular}{|c|c|c|c|}
\hline $\begin{array}{c}\text { Natural } \\
\text { frequencies }\end{array}$ & $\begin{array}{c}\text { Beam } \\
\text { structure }\end{array}$ & $\begin{array}{c}\text { Shell } \\
\text { structure }\end{array}$ & $\begin{array}{c}\text { ANSYS numerical } \\
\text { results }\end{array}$ \\
\hline$\omega_{1}, \mathrm{~Hz}$ & 0,7 & 0,64 & 0,63 \\
\hline$\omega_{2}, \mathrm{~Hz}$ & 4,4 & 3,58 & 3,8 \\
\hline$\omega_{3}, \mathrm{~Hz}$ & 12,32 & 9,82 & 10,18 \\
\hline$\omega_{4}, \mathrm{~Hz}$ & 24,16 & N/A & 18,83 \\
\hline$\omega_{5}, \mathrm{~Hz}$ & 39,96 & N/A & 29,19 \\
\hline
\end{tabular}

The results confirm that the described structure from Fig. 14 has a behavior of a shell.

\section{Conclusion}

In the present paper several important conclusions were arrived at:

1. The tower of NREL5MW wind turbine shows a behavior of a shell structure, not a beam and should be analyzed and considered as a shell.

2. The dynamic response of the structure under a stochastic generated wind excitations is dominated by the first mode of the structure, which represents nearly $98 \%$ of the response. Every subsequent mode contributes less, as compared to the previous one. The second and the third mode respectively represent only $2 \%$ and $0,2 \%$ of the response.

3. Due to the stochastic characteristics of the wind, the absence of correlation between the frequencies of the excitation forces, a resonance state does not appear. The structure passes through resonanse very fast and amplitudes of the forced vibrations do not increase.

\section{References}

1. J. Genov, I. Angelov, Influence of the vertical distribution of wind on the dynamic load of high-class wind turbines (in Bulgarian), Bultrans-2016 Proceedings, 136-140 (2016)

2. J. Jonkman, S. Butterfield, W. Musial, G. Scott, Definition of a $5 \mathrm{MW}$ reference wind turbine for offshore system development, NREL Technical report NRELTP-500-38060, 15-16 (2009)

3. J. Genov, B. Gilev, Y. Slavchev, G. Venkov, Modeling and control of wind turbine tower vibrations, Applications of Mathematics in Engineering and Economics - AMME-10, American Institute of Physics, Conference Proceedings 1293, 30-38 (2010)

4. M. Shinozuka, G. Deodatis, Simulation of stochastic processes by spectral representation, Applied Mechanics Review, 44, 191-204 (1991)

5. J. Wauer, Kontinuumsschwingungen, 92-97 (Springer Vieweg, 2008)

6. J. Tonchev, Matlab 6-7, Transformations, calculations, visualisations (in Bulgarian), 79-82 (Tehnika, Sofia, 2008)
7. S. Rao, Vibrations of continuous systems, 47-52; 556590 (John Wiley \& Sons Inc., 2007)

8. K. Forsberg, A review of analytical methods used to determine the modal characteristics of cylindrical shells, NASA Report Lockheed Aircraft Corporation, (1966)

9. G. Warburton, J. Higgs, Natural characteristics of thin cantilever cylindrical shells, Journal of Sound and Vibration, 11(3), 335-338 (1970)

10. C. Sharma, Calculation of natural frequencies of fixed-free circular cylindrical shell, Journal of Sound and Vibration, 35(1), 55-76 (1974) 